Pacific Journal of Mathematic 


\title{
ALGEBRAIC PROPERTIES OF CERTAIN RINGS OF CONTINUOUS FUNCTIONS
}

\author{
LI PI Su
}

Let $X$ and $Y$ be any subsets of $E^{n}$, and $\left(X^{\prime}, d_{1}\right)$ and $\left(Y^{\prime}, d_{2}\right)$ be any metric spaces. Let $C^{m}(X), 0 \leqq m \leqq \infty$, denote the ring of $m$-differentiable functions on $X$, and $L_{c}\left(X^{\prime}\right)$ be the ring of the functions which are Lipschitzian on each compact subset of $X^{\prime}$, and $L\left(X^{\prime}\right)$ be the ring of the bounded Lirschitzian functions on $X^{\prime}$. The relations between algebraic properies of $C^{m}(X)$, (resp. $L_{c}\left(X^{\prime}\right)$ or $L\left(X^{\prime}\right)$ and the topological properties of $X$ (resp. $\left.X^{\prime}\right)$ are studied. It is proved that if $X$ and $Y$, (resp. $\left(X^{\prime}, d_{1}\right)$ and $\left.\left(Y^{\prime}, d_{2}\right)\right)$ are $m$-realcompact, (resp. $L_{c}$-real-compact or compact) then $C^{m}(X) \cong C^{m}(Y)$ (resp. $L_{c}\left(X^{\prime}\right) \cong L_{c}\left(Y^{\prime}\right)$ or $L\left(X^{\prime}\right) \cong L\left(Y^{\prime}\right)$ if and only if $X$ and $Y$ are $C^{m}$-diffeomorphic (resp. $\left(X^{\prime}, d_{1}\right)$ and $\left(Y^{\prime}, d_{2}\right)$ are $L_{c}$ or $L$-homeomorphic).

During the last twenty years, the relations between the algebraic properties of $C^{m}(X)$ and $C^{m}(Y)$ and the topological properties of $X$ and $Y$ have been investigated by Hewitt [4], Myers [9], Pursell [11], Nakai [10], and Gillman and Jerison [3], where $m$ is a positive integer, zero or infinite. In 1963, Sherbert [12] studied the ring $L(X)$. Recently, Magill, [6] has obtained the algebraic condition relating $C(X)$ and $C(Y)$ (i. e., $m=0$ ) which are both necessary and sufficient for embedding $Y$ in $X$, where $X$ and $Y$ are two realcompact spaces.

This work is to utilize the method of Gillman and Jerison [3] for studying the algebraic properties of $C^{m}(X)$ and $L_{c}\left(X_{1}\right)(\S \S 2-5)$, and how they are related with topological properties of $X$ and $X_{1}$ respectively. In view of [8, Cor. 1.32], we will restrict $X$ in $C^{m}(X)$ to a subset of $E^{n}$. The results of Magill are also true in $C^{m}(X)$ and $L_{c}(X)$ with some modification. In the last section, $\S 6$, we observe some other cases.

2. Rings and ideals. Let $X$ be an arbitrary subset of $E^{n}$, an $n$-dimensional euclidean space, and $C^{m}(X)$ be the set of all real-valued functions of class $C^{m}$ in the sense of Whitney $[14, \S 3]$, where $m$ will always refer to an arbitrary integer such that $0 \leqq m \leqq \infty$. By [15, Th. 4], we know that $C^{m}(X)$ forms a ring with the identity $u$, the constant function of value 1 , and zero element $\theta$, the constant function of value 0 . Let $C^{m *}(X)=\left\{f \in C^{m}(X): f\right.$ is bounded $\}$. It is clear that $C^{m *}(X)$ is a subring of $C^{m}(X)$ with $u$ and $\theta$. Let $X$ be a metric space, and $L_{c}(X)$ be the set of all real-valued functions satisfying Lipschitz condition on each compact subset of $X$ [2, p. 354]. We can easily show that $L_{c}(X)$ is a ring with $u$ and $\theta$. Let $L(X)=$ 
$\left\{f \in L_{c}(X): f\right.$ is bounded and Lipschitzian on entire $\left.X\right\}, L_{c}^{*}(X)=$ $\left\{f \in L_{c}(X): f\right.$ is bounded $\}$. Then, both $L(X)$ and $L_{c}^{*}(X)$ are the subrings of $L_{c}(X)$ with $u$ and $\theta$.

Since the properties of $C^{m}(X)$ (resp. $\left.C^{m *}(X)\right)$ and those of $L_{c}(X)$ (resp. $L_{c}^{*}(X)$ and $L(X)$ ) are almost all the same, we will use $\mathfrak{U}$ and $\mathfrak{Y}^{\prime}$ to denote $C^{m}(X)$ (resp. $\left.L_{c}(X)\right)$ and $C^{m}(Y)$ (resp. $\left.L_{c}(Y)\right)$, and $\mathfrak{B}$ and $\mathfrak{B}^{\prime}$ to denote $C^{m *}(X)$ (resp. $L_{c}^{*}(X)$, and $L(X)$ ) and $C^{m *}(Y)$ (resp. $L_{c}^{*}(Y)$ and $\left.L(Y)\right)$ respectively, where $X$, and $Y$ are appropriately the subsets of $E^{n}$ or metric space. Also " $a$-" and " $b$-" will mean $m$ (or $C^{m_{-}}$) (resp. $L_{c}$ ) and $C^{m *}$ (resp. $L_{c}^{*}$ and $L$ ) respectively according as $\mathfrak{A}$ is $C^{m}(X)$ (resp. $L_{c}(X)$ ) and $\mathfrak{B}$ is $C^{m *}(X)$ (resp. $L_{c}^{*}(X)$, and $L(X))$.

The unit element of an $f \in \mathfrak{U}$ or $\mathfrak{B}$ is defined as usual. For $f \in \mathfrak{N}, Z(f)=\{x \in X: f(x)=0\}$ is said to be the zero-set of $f \cdot Z(\mathfrak{N})=$ $\{Z(f): f \in \mathfrak{A}\}$. It is then clear that $f \in \mathfrak{A}$ is a unit if and only if $Z(f)=\varnothing$. (For $C^{m}(X)$ see [15, Th. 4].) Likewise, if $f \in \mathfrak{B}$ is a unit, then $Z(f)=\varnothing$. But the converse need not hold, for the multiplicative inverse $1 / f$ of $f$ in $\mathfrak{A}$ may not be a bounded function. For example: let $X=E^{1}$, and $f(x)=e^{-x^{2}} \in C^{m *}\left(E^{1}\right)$ and $Z(f)=\varnothing$. But $1 / f=e^{x^{2}} \notin C^{m *}\left(E^{1}\right)$.

A $z$-filter of $Z(\mathfrak{U})$ is the same as in [3,2.2]. It is obvious that $Z[I]=\{Z(f): f \in I\}$ is a $z$-filter on $X$ if $I$ is a proper ideal in $\mathfrak{A}$, and $Z^{-1}[\mathscr{F}]=\{f \in \mathfrak{A}: Z(f) \in \mathscr{F}\}$ is a proper ideal if $\mathscr{F}$ is a $z$-filter on $X$. Note that it may be false that a proper ideal $I \subset \mathfrak{B}$ implies that $Z[I]$ is a $z$-filter. For example: let us consider $\mathfrak{B}=C^{m *}\left(E^{1}\right)$ and let $f(x)=1 /\left(1+x^{2}\right)$, and $I=(f)$ be the ideal generated by $f$ in $\mathfrak{B}$. Then it is clear that $\varnothing \in Z[I]$.

Hereafter, we will always use "ideal" to mean the proper ideal, unless the contrary is mentioned.

Accordingly, every $z$-filter is of the form $Z[I]$, for some ideal $I$ in $\mathfrak{A}$. That $Z^{-1}[Z[I]] \supset I$ is also clear. The inclusion may be proper.

For instance, consider $\mathfrak{U}=C^{m}\left(E^{1}\right)$. (a) For any positive integer $m$, let $i(x)=x$ for all $x \in E^{1}$, and $I=(i)$. Then

$$
Z^{-1}[Z[I]]=M_{0}=\left\{f \in C^{m}(E): f(0)=0\right\} .
$$

However, $i^{(3 m+1) / 3} \in M_{0}-I$. (b) In case $m=\infty$, let $f_{1}(x)=e^{-1 / x^{2}}$ for $x \in E^{1}$ and $I_{1}=\left(f_{1}\right)$. Then $M_{0}=Z^{-1}[Z[I]]$ contains an element $i \notin I_{1}$. Note that $M_{0}$ is a maximal fixed ideal. Now, as for $L_{c}(X)$, we may consider $(X, d)$ to be a bounded metric space, and $f_{0}(x)=\left(f_{p}(x)\right)^{2}=$ $(d(p, x))^{2}$. Then $f_{0} \in L_{c}(X)$. Let $I_{0}=\left(f_{0}\right)$. Then

$$
Z^{-1}\left[Z\left[I_{0}\right]\right]=\left\{f \in L_{c}(X): f(p)=0\right\}=M_{p}
$$

is clear. However, $f_{p}(x)=d(p, x) \in M_{p}-I_{0}$. 
A $z$-ultrafilter on $X$ is a maximal $z$-filter $[3,2.5]$. We know that every subfamily of $Z(\mathfrak{A})$ with the finite intersection property, by Zorn's Lemma, is contained in some $z$-ultrafilter on $X$.

The proofs of following propositions are obvious.

Proposition 2.1. If $M$ is a maximal ideal in $\mathfrak{A}$, then $Z[M]$ is a $z$-ultrafilter on $X$.

Proposition 2.2. If $\mathscr{A}$ is a $z$-ultrafilter on $X$, then $Z^{-1}[\mathscr{A}]$ is a maximal ideal in $\mathfrak{A}$.

It follows from Propositions (2.1) and (2.2) that the mapping $Z$ is one-one from the set of all maximal ideals in $\mathfrak{A}$ onto the set of all $z$-ultrafilters on $X$.

Proposition 2.3. Let $M$ be a maximal ideal in $\mathfrak{A}$. If $Z(f)$ meets every member of $Z[M]$, then $f \in M$.

Proposition 2.4. Let $\mathscr{A}$ be a $z$-ultrafilter on $X$. If a zero-set $Z$ meets every member of $\mathscr{A}$, then $Z \in \mathscr{A}$.

An ideal $I$ in $\mathfrak{A}$ is $z$-ideal if $Z(f) \in Z[I]$ implies $f \in I$. That is, $I=Z^{-1}[Z[I]],[3,2.7]$. It is obvious that every maximal ideal is a $z$ ideal. A prime ideal is defined in the usual sense. The following theorem is only true for $L_{c}(X), L_{c}^{*}(X)$ or $L(X)$. For we can show that these are lattice-ordered rings; while $C^{m}(X)$ and $C^{m *}(X)$ are not.

Theorem 2.5. For any z-ideal $I$ in $L_{c}(X)\left(L_{c}^{*}(X)\right.$ or $\left.L(X)\right)$ the following are equivalent:

(1) I is prime.

(2) I contains a prime ideal.

(3) For all $g, h \in L_{c}(X)\left(L_{c}^{*}(X)\right.$ or $\left.L(X)\right), g \cdot h=\theta$, then $g \in I$ or $h \in I$.

(4) For every $f \in L_{c}(X)\left(L_{c}^{*}(X)\right.$ or $\left.L(X)\right)$, there is a zero-set in $Z[I]$ on which $f$ does not change sign.

Proof is similar to $[3,2.9]$.

3. Zero-set, $a$-completely regular and $a$-normal spaces. We know from the proof of Lemma $25[16$, p. 669] that each closed subset $F$ of $E^{n}$, there is an $f \in C^{m}(X)$ such that $Z(f)=F$.

Proposition 3.1. For each closed subset $A$ of $(X, d)$, there is $f \in L_{c}(X)$ (in fact $f \in L(X)$ ) such that $Z(f)=A$. 
Proof. Let $g(x)=d(A, x)$ and $f=g \wedge u^{1}$. Then $f \in \mathrm{L}(X)$ and $Z(f)=A$.

Definition 3.2. Let $X$ be a topological space. $X$ is said to be $a$-completely regular if and only if for each closed subset $F$ of $X$ and $x \notin F$, there is an $f \in \mathfrak{A}$ such that $f(x)=1$, and $f[F]=\{0\}$.

THEOREM 3.3. A topological space is a-completely regular if and only if the family $Z(\mathfrak{H})=\{Z(f): f \in \mathfrak{U}\}$ is a base for the closed subsets of $X$.

Proof is similar to $[3,3.2]$.

Definition 3.4. A topological space is said to be $a$-normal if for any disjoint closed subsets $F_{1}$ and $F_{2}$, there is an $f \in \mathfrak{A}$ such that $f\left[F_{1}\right]=\{0\}$ and $f\left[F_{2}\right]=\{1\}$.

Proposition 3.5. Every subset $X$ of $E^{n}$ is $m$-normal. Hence is m-completely regular.

Proof. Let $F_{1}$ and $F_{2}$ be any two disjoint closed subsets of $X$. We know that there are closed subsets $F_{1}^{\prime}$ and $F_{2}^{\prime}$ of $E^{n}$ such that $F_{i}=F_{i}^{\prime} \cap X, i=1,2$. We know that there are $f_{i} \in C^{m}\left(E^{n}\right)$ with $Z\left(f_{i}\right)=F_{i}^{\prime}$. Let $g_{i}=f_{i} \mid X$, and $f=g_{1}^{2} /\left(g_{1}^{2}+g_{2}^{2}\right)$. Then $f \in C^{m}(X)$ and $Z(f)=Z\left(g_{1}\right)=F_{1}, f\left[\mathrm{~F}_{2}\right]=\{1\}$. The last part is obvious.

Proposition 3.5'. Every metric space $(X, d)$ is $L$-completely regular; and every compact metric space $(X, d)$ is $L$-normal.

Proof. Let $F$ be a closed subset of $X$ and $p \in X-F$. Then $d(F, p) \neq 0$. Let $f$ be defined as follows: $f[F]=\{1\}$, and $f(p)=0$. Then $f$ is bounded by 1 satisfies a Lipschitz condition with constant $K=(d(F, p))^{-1}$ on $F \cup\{p\}$. We know that there exists $f_{0} \in L(X)$ such that $f_{0} \mid F \cup\{p\}=f$, (by [7, p. 97]). Hence the first assertion follows. The proof of the second part is the same.

Note that the compactness in $(3.5)^{\prime}$ cannot be omitted. For instance: let $X=E^{2}$,

$$
F=\left\{(x, y) \in E^{2}: x y=1\right\} \text { and } F^{\prime}=\left\{(x, y) \in E^{2}: x y=-1\right\} .
$$

Then $F$ and $F^{\prime}$ are two disjoint closed sets in $E^{2}$. However, it is clear that there is no $f \in L\left(E^{2}\right)$ such that $f[F]=\{1\}$ and $f\left[F^{\prime}\right]=\{0\}$.

\footnotetext{
${ }^{1} u$ stands for the constant function of value 1 .
} 
Having done these, we can show the characterization of fixed maximal ideal of $\mathfrak{A}$ and $\mathscr{B}$ and how they are related to a compact space are the same as in [3, (4.6), (4.8), (4.9) (a), (4.10) Lemma, and (4.11)].

4. Real ideals, $a$-realcompact space. In 1948, E. Hewitt defined real maximal ideals and realcompact space ( $Q$-spaces) (see $[4, \S 7]$ and [3, Ch. 5]). He also contributed many interesting properties about real maximal ideals and realcompact spaces. Unfortunately, those properties can only be carried to the rings $L_{c}(X), L_{c}^{*}(X)$ and $L(X)$, but not to $C^{m}(X)$, since $C^{m}(X)$ is not a lattice-ordered ring. (As $f \in C^{m}(X)$ implies $|f| \in C^{m}(X)$ is not always true.) Recently (1965), Rudolphe Bkouche has shown that every paracompact Hausdorff differentiable $n$-manifold is $m$-realcompact (see (4.2), and [1, Th. 2]). Here will show that every closed subset of $E^{n}$ is $m$-realcompact.

We can show easily that every residue class field of $\mathfrak{U}$ or $\mathscr{B}$ module a maximal ideal contains canonical copy of real field $\boldsymbol{R}$. We can also show, by using [3, (5.1) to (5.4)] or [13], that $L_{c} / M, L_{c}^{*} / M$, and $L(X) / M$ are totally ordered for each maximal ideal $M$. We will show that $L_{c}^{*}(X) / M$ (resp. $\left.L(X) / M\right) \cong \boldsymbol{R}$ if $M$ is maximal in $L_{c}^{*}$ (resp. $L(X))$. The real and hyper-real ideals are defined in $[3,5.9]$.

Lemma 4.1. Let $M$ be a maximal ideal in $L_{c}^{*}(X)$, (resp. $\left.L(X)\right)$, and $L_{c}^{*}(X)$, (resp. $\left.L(X)\right)$ be normed by the sup norm $\|\cdot\|_{\infty}$. Then $M$ is closed in $L_{c}^{*}(X)$ (resp. $\left.L(X)\right)$ under $\|\cdot\|_{\infty}$.

Proof. In view of [3,2 Ml], cl $M$ is either a proper ideal of $L_{c}^{*}(X)$ or $L_{c}^{*}(X)$ itself. Suppose $\operatorname{cl} M=L_{c}^{*}(X)$. Then $u \in \operatorname{cl} M$, and for any neighborhood of $u, N_{\varepsilon}(u), N_{\varepsilon}(u) \cap M \neq \varnothing$. Take $\varepsilon=1 / 2$. Then $N_{1 / 2}(u) \cap M \neq \varnothing$. That is, there is an $f \in M$ such that

$$
\|u-f\|_{\infty}<\frac{1}{2}
$$

This implies $|f(x)|>1 / 2$ for each $x \in X$. We can easily show that $1 / f \in L_{c}^{*}(X)$. That is, $M$ has a unit so that $M=L_{c}^{*}(X)$. This is a contradiction. Hence cl $M$ is a proper ideal containing $M$ so that $M=\operatorname{cl} M$. The proof for $M$ in $L(X)$ is similar.

Proposition 4.2. For each maximal ideal $M$ in $L_{c}^{*}(X)$, (resp. $L(X)), L_{c}^{*}(X) / M(\operatorname{resp} . L(X) / M) \cong \boldsymbol{R}$.

Proof. It is enough to show that for any positive nonconstant residue class $M(f)$, simply denoted by $f$, there is a positive integer $n$ such that $f-1 / n$ is positive. (See [3,5.6] and [13].) Suppose that 
there does not exist such a positive integer. Then we would have $f-1 / n$ is negative for all $n \in N$. That is, $(f-1 / n)+|f-1 / n| \in M$ for all $n$. Consider now the sequence $\left\{g_{n}=(f-1 / n)+f-1 / n: n \in N\right\}$ which has $f+|f|$ as the limit under the norm $\|\cdot\|_{\infty}$. By (4.1), $f+|f| \in \operatorname{cl} M=M$. This shows that $-f \equiv|f|(\bmod M)$. This is a contradiction.

Definition 4.3. A topological space $X$ is said to be $a$-realcompact if every real maximal ideal in $\mathfrak{A}$ is fixed.

It is clear that if $X$ is compact, then $X$ is $a$-realcompact.

Lemma 4.4. An ideal in $\mathfrak{U}$ is free if and only if for every compact subset $A$ of $X$ there exists an $f \in I$ having no zero in $A$.

Proof. Suppose $I$ is free and $A$ is any compact subset of $X$. If for each $f \in I, Z(f) \cap A \neq \varnothing$, then $\mathscr{F}=\{Z(f) \cap A$ : for some $f \in I\}$ has the finite intersection property. Since $A$ is compact, $\cap \mathscr{F} \neq \varnothing$. Hence $\cap Z[I] \supseteqq \cap \mathscr{F} \neq \varnothing$, which is impossible.

The sufficiency is clear.

Proposition 4.5. Let $X$ be a closed subspace of $E^{n}$. Then $X$ is $m$-realcompact as well as $L_{c}$-realcompact.

Proof. Suppose that $M$ is a free maximal ideal and $C^{m}(X) / M \cong \boldsymbol{R}$. Let $g(x)=1 /\left(\|x\|^{2}+1\right)$. Then that $g \in C^{m}(X)$ and $g$ is a unit is clear. Hence $g \notin M$. i.e., $M(g) \neq 0$. For any positive number $r$ and a sufficiently small number $\varepsilon>0, g<r-\varepsilon$ for all but a compact subset of $E^{n}$, say $A_{\varepsilon}$. Then $B_{\varepsilon}=A_{\varepsilon} \cap X$ is compact in $X$ as $X$ is closed. Let $A^{\prime}=\operatorname{cl}_{X}\left(X-B_{\varepsilon}\right)$. Then there is an $f \in C^{m}(X)$ such that $Z(f)=A^{\prime}$. We will show that $Z(f) \in Z[M]$. We know that $B_{\varepsilon}$ is compact in $X$. By (4.4), there is an $f_{1} \in M$ such that $Z\left(f_{1}\right) \cap B_{\varepsilon}=\varnothing$. Hence $Z\left(f_{1}\right) \leqq$ $X-B_{\varepsilon} \leqq Z(f)$ so that $Z(f) \in Z(M)$. Therefore, $g \leqq r-\varepsilon$ on the zeroset $Z(f)$, and $r-g \geqq \varepsilon$. Let $h_{1}=(r-g)^{1 / 2}$ on $Z(f)$. Then $h_{1}$ is $C^{m}$ on $Z(f)$ which is closed in $E^{n}$. By Whitney's Analytic Extension Theorem [14], we have a $C^{m}$ extension $h$, i.e., $h \mid Z(f)=h_{1}$. Hence $h^{2}=r-g$ on $Z(f)$. Therefore, $h^{2} \equiv r-g(\bmod M)$. In other words, $M\left(h^{2}\right)=M(r-g)=M(r)-M(g)=r-M(g)$. But, since $C^{m}(X) / M$ is real $M\left(h^{2}\right) \geqq 0$, we have $M(g) \leqq r$. As $r$ is any positive number, $M(g)$ is infinitely small. This is a contradiction. The proof of the last part is similar.

We now will give an example to show that a nonparacompact space may not be an $m$-realcompact space. However, the existence of non- $L_{e}$-realcompact spaces remains as an open question.

Let $L$ be the long line as defined in [5]. Then, $L$ is Hausdorff 
space satisfying the first axiom of countability. Furthermore we have:

Proposition 4.6. For each $\alpha \in L, \alpha \neq 1,[1, \alpha]$ is isotonehomeomorphic to unit interval $[0,1]$. Consequently, each point of $L$, not the first element, 1, has an open neighborhood which is homeomorphic to an open interval.

Proof. Use transinfinite induction.

Proposition 4.7. $L$ is countably compact but is not paracompact, hence is not a compact space.

Proof. Let $A$ be any countably infinite subset of $L$. Then, $A$ will be contained in the union of $\left\{I_{\alpha}: \alpha \in \Delta \subset W\right\}$, where $W$ is the set of all ordinal numbers less than the first uncountable ordinal, and $\Delta$ has at most countably many elements. Let $\alpha_{0}$ be the least upper bound of $\Delta$. Then $\left[1, \alpha_{0}\right]$ is homeomorphic to [0,1]. Hence $A \subset\left[1, \alpha_{0}\right]$, a compact set, must have a limit point in $\left[1, \alpha_{0}\right] \subset L$, so that $L$ is countably compact. In view of $(4.6), L$ is locally metrizable [5, p. 80] but $L$ is not metrizable. By Theorem 2-68 [5, p. 81] $L$ is not paracompact. Hence, it is not compact.

Proposition 4.8. Of any two disjoint closed sets in $L$, one is bounded.

Proof is similar $[3,5.12(b)]$.

By (4.6), we know that $L$ is a 1-dimensional manifold with a boundary point 1 . Hence we may define the differentiable function on $L$.

Proposition 4.9. Every function $f \in C(L)$ is a constant on a tail $L-L(\alpha)$ where $\alpha$ depends on $f$, and $L(\alpha)=\{\sigma \in L: \sigma<\alpha\}$.

Proof is similar to $[3,5.12(\mathrm{c})]$.

Let $L^{*}$ be the union space of $L$ and the point $\Omega$, the first uncountable ordinal. Then, $L^{*}$ is a compact 1-dimensional manifold. For each $f \in C^{m}(L)$, we extend $f$ to a function $f^{*}$ on $L^{*}$ by defining that $f^{*}(\Omega)$ is the final constant value of $f$. Evidently $f^{*} \in C^{m}\left(L^{*}\right)$ and is unique. On the other hand, for each $g \in C^{m}\left(L^{*}\right), g \mid L \in C^{m}(L)$. Hence $C^{m}(L)$ is isomorphic with $C^{m}\left(L^{*}\right)$, under the mapping $f \rightarrow f^{*}$.

Since $L^{*}$ is compact, every ideal is fixed, and the maximal ideals assume the form $M_{\sigma}=\left\{f^{*} \in C^{m}\left(L^{*}\right): f^{*}(\sigma)=0\right\}$, where $\sigma \in L^{*}$. By virtue of isomorphism of $C^{m}\left(L^{*}\right)$ with $C^{m}(L)$, the maximal ideals in $C^{m}(L)$ are in one-one correspondence with those of $C^{m}\left(L^{*}\right)$. Moreover, 
the fixed maximal ideals in $C^{m}(L)$ correspond to the ideals $M_{\sigma}$ in $C^{m}\left(L^{*}\right)$ for each $\sigma \in L$, leaving just one free maximal ideal in $C^{m}(L)$, namely, $M_{0}=\left\{f \in C^{m}(L): f^{*} \in M_{\Omega}\right\}$, the one that corresponds to $M_{\Omega}$. Though $M_{0}$ is free, it is not hyper-real, for $C^{m}(L) / M_{0} \cong C^{m}\left(L^{*}\right) / M \cong \boldsymbol{R}$. Hence $L$ is not $m$-realcompact.

5. Homomorphism, $a$-mapping and $a$-homeomorphism. In this section we will describe the relation between any $a$-mapping from $X$ into $Y$ and homomorphisms from $\mathfrak{X}^{\prime}$ to $\mathfrak{A}$.

Definition 5.1. Let $X \subset E^{n_{1}}$, and $Y \subset E^{n_{2}}$. A mapping $\tau: X \rightarrow Y$ is said to be a $C^{m}$-mapping at a point $p$, if each component of $\tau(x)=$ $\left(\tau_{1}\left(x_{1}, \cdots, x_{n_{1}}\right), \cdots, \tau_{n_{2}}\left(x_{1}, \cdots, x_{n_{1}}\right)\right)$ is $C^{m}[14, \S 3]$ at $p$. If $\tau$ is $C^{m}$ at each point of $X$, then $\tau$ is said to be a $C^{m}$-mapping on $X$. If $\tau$ is a $C^{m}$-mapping, one-one, onto $Y$ and its inverse mapping, $\tau^{-1}$, is also a $C^{m}$-mapping, then $\tau$ is $C^{m}$-diffeomorphism. We will say then $X$ and $Y$ are $C^{m}$-diffeomorphic.

Note that by (5.1), $X$ and $Y$ are $C^{m}$-diffeomorphic implies $n_{1}=n_{2}$.

Difinition 5.1.' A mapping $\tau$ from $\left(X, d_{1}\right)$ to $\left(X, d_{2}\right)$ is said to be an $L_{c}$-(resp. $L$-) mapping if, for each compact subset $A$ of $X$, there is a positive number $K_{A}$ such that $d_{2}\left(\tau(x), \tau\left(x^{\prime}\right)\right) \leqq K_{A} d_{1}\left(x, x^{\prime}\right)$ for all $x, x^{\prime} \in A$. (resp. if there is a positive number $K$ such that

$$
d_{2}\left(\tau(x), \tau\left(x^{\prime}\right)\right) \leqq K d\left(x, x^{\prime}\right)
$$

for all $\left.x, x^{\prime} \in X\right) . \quad \tau$ is said to be an $L_{c}$-(resp. $L$-) homeomorphism, if $\tau$ is one-one, onto $Y$ and both $\tau$ and its inverse $\tau^{-1}$ are $L_{c}$-(resp. $L$-) mappings.

We will use " $a$-mapping" to mean $C^{m}$-mapping, $L_{c}$-or $L$-mapping, and " $a$-homeomorphism" to mean $C^{m}$-diffeomorphism, $L_{c}$ - or $L$ homeomorphism according as $\mathfrak{A}$ is $C^{m}(X), L_{c}(X)$ or $\mathscr{B}=L(X)$.

Definition 5.2. An $f \in C^{m}(X)$ is said to be a local $i$-th projection at a point $p$ if there exists a neighborhood $U$ of $p$ such that $f \mid U=i$, where $i$ always denotes the $i$-th projection of the space $E^{n}$ or $X \subset E^{n}$.

Lemma 5.3. Let $X$ be any subset of $E^{n}$. For each $p \in X$ and $r>0$, there are $h_{i},(1 \leqq i \leqq n), h_{i} \in C^{\infty *}(X)$ such that $h_{i}(x)=x_{i}$ for all $x \in \operatorname{cl}_{X} B_{r}(p)$. We call $h_{i},(1 \leqq i \leqq n)$ the $i$-th bounded local projection at $p$.

Proof. Choose $r^{\prime}>r$. It is well-known that there exists $g \in C^{\infty *}\left(E^{n}\right)$ such that 


$$
g(x)=\left\{\begin{array}{l}
1 \text { if } x \in \operatorname{cl}_{E}{ }^{n} B_{r}(p) \\
0 \text { if } x \in E^{n}-B_{r^{\prime}}(p) \\
0<g(x)<1, \text { elsewhere }
\end{array}\right.
$$

Set $h_{i}(x)=i(x) \cdot g(x)$.

Let $C_{0}^{m}$ be a subset of $C^{m}(Y)$ (resp. $C^{m *}(Y)$ ), and $\tau$ be a mapping from $X$ to $Y$. Then we will see what $C_{0}^{m}$ should be in order that $g \cdot \tau \in C^{m}(X)$ (resp. $C^{m *}(X)$ ) for all $g \in C_{0}^{m}$ implies $\tau$ is a $C^{m}$-mapping from $X$ into $Y$.

Theorem 5.4. Let $\tau$ be a mapping from $X$ to $Y$ and $C_{0}^{m}$ be a subset of $C^{m}(Y)$.

(1) $\tau$ is a $C^{m}$-mapping implies $g \cdot \tau \in C^{m}(x)$ for all $g \in C_{0}^{m}$.

(2) If $g \cdot \tau \in C^{m}(X)$ for each $g \in C_{0}^{m}$, and $C_{0}^{m}$ includes all projections of $X$, then $\tau$ is a $C^{m}$-mapping on $X$.

Proof. (1) It is obvious. (2) Since $g \cdot \tau \in C^{m}(X)$ for each $g \in C_{0}^{m}$ which includes all projections on $X$, we have, in particular, $i \cdot \tau(x)=$ $\tau_{i}(x) \in C^{m}(X)$ for $1 \leqq i \leqq n_{2}$. Hence, by (5.1) $\tau$ is a $C^{m}$-mapping.

THEOREM 5.4.* Let $\tau$ be a mapping from $X$ to $Y$ and $C_{0}^{m}$ be a subset of $C^{m *}(Y)$.

(1) $\tau$ is $C^{m}$-mapping implies $g \cdot \tau \in C^{m *}(X)$ for all $g \in C_{0}^{m}$,

(2) If $g \cdot \tau \in C^{m}(X)$ for each $g \in C_{0}^{m}$, and $C_{0}^{m}$ includes all local projections, then $\tau$ is a $C^{m}$-mapping on $X$.

The proof is similar to (5.4)

THEOREM 5.4.' Let $\tau$ be a mapping from a metric space $\left(X, d_{1}\right)$ to another metric space $\left(Y, d_{2}\right)$.

(1) If $\tau$ is an $L_{c}$-mapping, then $f \cdot \tau \in L_{c}(X)$ for all $f \in L_{c}(Y)$.

(2) If $f \cdot \tau \in L_{c}(X)$ for all $f \in L_{c}(Y)$, then $\tau$ is an $L_{c}$-mapping of $\left(X, d_{1}\right)$ into $\left(Y, d_{2}\right)$.

Proof. (1) is clear. (2) Consider any compact subset $A \neq \varnothing$ of $X$. We will show that $\tau$ is an $L$-mapping on $A$. By [3,3.8] we know $\tau$ is continuous. Hence $\tau[A]$ is compact. Let $\Phi$ be a mapping from $L_{c}(Y)$ to $L_{c}(X)$ defined by $\Phi(f)=f \cdot \tau$ for all $f \in L_{c}(Y)$. Then, it is obvious that $\Phi$ is a homeomorphism of $L_{c}(Y)$ into $L_{c}(X)$. We restrict $\Phi$ to $L_{c}(Y) \mid \tau[A]=\left\{f \mid \tau[A]: f \in L_{c}(Y)\right\}$, then $\Phi$ is into

$$
L_{c}(X) \mid A=\left\{g \mid A: g \in L_{c}(X)\right\} \text {. }
$$

By compactness of $A$ and the fact that every function which is Lip- 
schitzian on a nonempty subset of a space can be extended to the whole space [7], we can show that $L_{c}(X) \mid A=L(A)$, and

$$
L_{c}(Y) \mid \tau[A]=L(\tau[A]) .
$$

By $[12,5.1] \tau$ is an $L$-mapping on $A$. Since $A$ is arbitrary, $\tau$ is an $L_{c}$-mapping.

The induced mapping is defined in $[3,10.2]$. We are concerned with an $a$-mapping $\tau$ of $X$ into $Y$, where the role of $D$ in $[3,10.2]$ is taken by $E^{1}$. The appropriate subset of $E^{1^{Y}}$ will be $\mathfrak{U}^{\prime}$ or $\mathscr{B}^{\prime}$. Evidently, the induced mapping $\tau^{\prime}$, defined by $\tau^{\prime}(g)=g \cdot \tau \in \mathfrak{A}$ for each $g \in \mathfrak{U}^{\prime}$ (resp. $\mathscr{B}^{\prime}$ ) is a homomorphism from $\mathfrak{Y}^{\prime}$ to $\mathfrak{X}$ (resp. $\mathscr{B}^{\prime}$ into $\mathscr{B}$ ), and $\tau$ carries the constant functions onto the constant functions identically. Moreover, $\tau^{\prime}$ determines the mapping $\tau$ uniquely.

We now examine the duality relation between $\tau$ and $\tau^{\prime}$.

Definition 5.5. A subset $A$ of $X \subset E^{n}$ is $C^{m}$ (resp. $C^{m *}$ )-embedded in $X$ if for each $f \in C^{m}(A)$ (resp. $C^{m *}(A)$ ) there is $g \in C^{m}(X)$ (resp. $\left.C^{m *}(X)\right)$ such that $g \mid A=f$.

Definition 5.5.' A subset $A$ of a metric space $(X, d)$ is $L_{c}$ (resp. $L_{c}^{*}$, or $L$ )-embedded in $X$ if for each $f \in L_{c}(A)$ (resp. $L_{c}^{*}(A)$, or $L(A)$ ), there is $g \in L_{c}(X)$ (resp. $L_{c}^{*}(X)$, or $L(X)$ ) such that $g \mid A=f$.

We will simply say that a subset $A$ of a topological space is $a$ (resp. b)-embedded if $A$ is $C^{m}$, or $L_{c}$ (resp. $C^{m *}, L_{c}^{*}$, or $L$ )-embedded.

THEOREM 5.6. Let $\tau$ be an a-mapping from $X$ into $Y$, and $\tau^{\prime}$

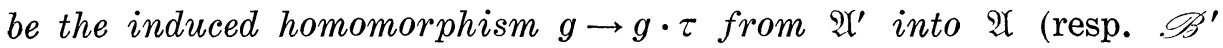
into $\mathscr{B})$.

(1) $\tau^{\prime}$ is an isomorphism (into) if and only if $\tau[X]$ is dense in $Y$.

(2) $\tau^{\prime}$ is onto if and only if $\tau$ is an a-homeomorphism whose image is a (resp. b)-embedded.

Proof. Having (5.4), (5.4)* (5.4) and (5.3) in hand, the proof is similar to $[3,10.3]$.

CoRollary 5.7. If $\tau$ is an a-homeomorphism from $X$ onto $Y$, then $\tau^{\prime}$ is an isomorphsim of $\mathfrak{U}^{\prime}$ onto $\mathfrak{A}$.

COROLLARY 5.8. If $\tau$ is an a-homeomorphism of a compact space $X$ to $Y$, then the induced mapping $\tau^{\prime}$ is onto. 
Proof. Use the theorem of Whitney's analytic extension [14] and the fact that each Lipschitzian function on a subset can be extended to the whole space [7]. The proof is evident.

Next, we examine the inverse problem of determining when a given homomorphism of $\mathfrak{A}^{\prime}$ into $\mathfrak{A}$ is induced by some $a$-mapping from $X$ into $Y$. We shall first consider the homomorphism from $\mathfrak{A}$ into $\boldsymbol{R}$, i.e., the case in which $X$ consists of just one point.

Proposition 5.9. Any nonzero homomorphism $\Phi$ from $\mathfrak{X}^{\prime}\left(\right.$ or $\left.\mathscr{B}^{\prime}\right)$ into $\boldsymbol{R}$ is onto $\boldsymbol{R}$. In fact $\Phi(r)=r$ for all $r \in \boldsymbol{R}$.

Proof is similar to $[3,10.5(a)]$.

Proposition 5.10. The correspondence between the homomorphism of $\mathfrak{U}^{\prime}\left(\right.$ or $\left.\mathscr{B}^{\prime}\right)$ onto $\boldsymbol{R}$, and the real maximal ideals is one-one.

Proof is similar to $[3,10.5(b)]$.

Proposition 5.11. $Y$ is a-realcompact if and only if to each nonzero homomorphism $\Phi$ from $\mathfrak{A}$ onto $\boldsymbol{R}$, there corresponds a unique point $y$ of $Y$ such that $\Phi(g)=g(y)$ for all $g \in \mathfrak{U}^{\prime}$.

Proof. Use (5.10) and $a$-realcompactness.

Our first result about homomorphisms from $\mathfrak{U}^{\prime}$ into $\mathfrak{A}$ for $X$ is a generalization of (5.11).

THEOREM 5.12. Let $\Phi$ be a homomorphism from $\mathfrak{X}^{\prime}$ into $\mathfrak{A}$ such that $\Phi(u)=u$. If $Y$ is a-realcompact, then there exists a unique a-mapping $\tau$ of $X$ into $Y$ such that $\tau^{\prime}=\Phi$.

Notice that the condition $\Phi(u)=u$ is necessary. Proof of the theorem is similar to $[3,10.6]$.

CoROLLARY 5.13. An a-realcompact space $Y$ contains an image of an a-mapping of $X$ if and only if $\mathfrak{A}$ contains a homomorphic image of $\mathfrak{Y}^{\prime}$ that included the constant functions on $X$.

Proof is similar to $[3,10.9(\mathrm{a})]$.

CoROLlary 5.14. An a-realcompact space $Y$ contains an image of an a-mapping which is dense in $Y$ if and only if $\mathfrak{A}$ contains an isomorphic image of $\mathfrak{U}^{\prime}$ that includes the constant functions on $X$. 
Proof is similar to $[3,10.9(\mathrm{~b})]$.

The MAIN Theorem. Two a-realcompact spaces $X$ and $Y$ are a-homeomorphic if and only if $\mathfrak{A}$ and $\mathfrak{U}^{\prime}$ are isomorphic.

Proof. The necessity follows from (5.7).

Sufficiency. Let $\Phi$ be an isomorphism of $\mathfrak{U}^{\prime}$ onto $\mathfrak{A}$. Then $\Phi^{-1}$ is an isomorphism of $\mathfrak{U}$ onto $\mathfrak{U}^{\prime}$. By (5.12), there exist unique $a$-mappings $\tau$ and $\tau_{1}$ from $X$ into $Y$ and from $Y$ into $X$, respectively, such that $\Phi(g)=g \cdot \tau$, and $\Phi^{-1}(f)=f \cdot \tau_{1}$, for each $g \in \mathfrak{Y} \mathfrak{Y}^{\prime}$ and $f \in \mathfrak{A}$. Then, $g(y)=\Phi^{-1}(g \cdot \tau)(y)=(g \cdot \tau) \cdot \tau_{1}(y)=g \cdot\left(\tau \cdot \tau_{1}\right)(y)$ for all $y \in Y$. That is, $\tau \cdot \tau_{1}$ is the identity mapping of $Y$ onto itself. Similarly, $\tau_{1} \cdot \tau$ is the identity mapping of $X$ onto itself. Thus $\tau$ and $\tau_{1}$ are the inverse mappings of each other. Hence $X$ and $Y$ are $a$-homeomorphic.

Remark. S.B. Myers [9], L.E. Pursell [11], and M. Nakai [10] have dealt with $C^{m}$-differentiable $n$-manifolds. This theorem is applicable to any closed subset of $E^{n}$.

In spite of the remark made in (5.12), every homomorphism is induced, in essence, by an $a$-mapping.

Theorem 5.16. Let $\Phi$ be a homomorphism from $\mathfrak{X}^{\prime}$ (resp. $\mathscr{B}^{\prime}$ ) into $\mathfrak{A}$ (resp. $\mathscr{B}), Y$ be a-realcompact (resp. compact). Then the set $E=\{x \in X: \Phi(u)(x)=1\}$ is open-and-closed in $X$. Moreover, there exists a unique a-mapping $\tau$ from $E$ into $Y$, such that for any $g \in \mathfrak{H}^{\prime}$ (resp. $\mathscr{B}) \quad \Phi(g)(x)=g(\tau(x))$ for all $x \in E$, and $\Phi(g)(x)=0$ for all $x \in X-E$.

Proof is similar to $[3,10.8]$.

COROLlaRY 5.17. Let $\Phi$ be a homomorphism from $\mathfrak{H}^{\prime}$ into a subring $R$ of $\mathfrak{A}$. If $Y$ is a-realcompact, then there exists a unique closed subset $F$ of $Y$ such that the kernel of $\Phi$ is the $z$-ideal of all functions in $R$ that vanish on $F$.

Proof. Let $E=\{x \in X: \Phi(u)(x)=1\}$. By (5.16), there exists an $a$-mapping $\tau$ from $E$ into $Y$ such that $\Phi(g)(x)=g(\tau(x))$ for all $x \in E$, and $\Phi(g)(x)=0$ for all $x \in X-E$, for all $g \in \mathfrak{Y ^ { \prime }}$. Let $F=\operatorname{cl}_{Y} \tau[E]$, and $I=\left\{g \in \mathfrak{U}^{\prime}: Z(g) \supseteqq F\right\}$. We can show easily that $\operatorname{ker} \Phi=I$. The uniqueness of $F$ is clear.

Proposition 5.18. An $a$-realcompact (resp. compact) space $Y$ contains an image of an $a$-mapping of $X$ which is a (resp. b)-embedded 
if and only if $\mathfrak{A}($ resp. $\mathscr{B})$ is a homomorphism image of $\mathfrak{A}^{\prime}$ (resp. $\mathscr{B}^{\prime}$ ).

Proof is similar to $[3,10.9(\mathrm{c})]$.

REMARK. With the previous results, one can show without any difficulty that the Theorems (2.1), (2.3) (2.4), (2.5) and (2.6) of [6] are true if $C(X)$ is replaced by $C^{m}(X)$ (resp. $L_{c}(X)$ ) with the condition that $\Phi\left(C^{m}(X)\right)$ contains all projections or all local projections of $C^{m}(Y)$ (resp. $\left.\Phi\left(L_{c}(X)\right)=L_{c}(Y)\right)$. However, in Theorem (2.2) [6], only the first three statements are equivalent. For $\Phi$ is not a lattice homomorphism (see $[3,0.5]$ ) from $C^{m}(Y)$ to $C^{m}(X)$ (resp. $L_{c}(Y)$ to $\left.L_{c}(X)\right)$.

6. Remarks. We have shown that if $X$ and $Y$ are two $a$-realcompact spaces, then $\mathfrak{A}$ and $\mathfrak{Y}^{\prime}$ are isomorphic if and only if $X$ and $Y$ are $a$-homeomorphic. We shall make some observations about other cases.

Let $X$ be a subset of $E^{n}$, and $S_{1}$ be the set of the projections and the constant functions on $X$. Let $S_{2}$ be the ring generated by $S_{1}$, and $\mathscr{R}(X)=\left\{f / g: f, g \in S_{2}\right.$ and $\left.Z(g)=\varnothing\right\}$. Evidently $\mathscr{R}(X)$ is a commutative ring of rational functions on $X$ with unity $u$ and zero element $\theta$. A ring of functions, $A(X)$, is said to satisfy property $(6-1)$, if $\mathscr{R}(X) \subseteq A(X) \subseteq C^{m}(X)$, and if $f \in A(X)$ with $Z(f)=\varnothing$, then $1 / f \in A(X)$.

Lemma 6.1. If $A(X)$ satisfies property (6-1), then there is an $f \in A(X)$ such that $f$ belongs to no maximal ideal other than $M_{a}=$ $\{f \in A(X): f(a)=0\}$ and $Z(f)=\{a\}$, for each $a \in X$.

Proof. Take $f(x)=\sum_{i=1}^{n}\left(x_{i}-a_{i}\right)^{2}$, where $\left(a_{1}, \cdots, a_{n}\right)=a \in X$. Then that $f \in M_{a}$ and belongs to no other fixed maximal ideal is clear. If $f$ belongs to a free maximal ideal $M$, then there is $g \in M$ with $g(a) \neq 0$. Let $h=f^{2}+g^{2}$. We have $Z(h)=\varnothing$ so that $1 / h \in A(X)$. Hence $u=h h^{-1} \in M$. This is impossible.

Lemma 6.1.' For any metric space $(X, d)$ and $p \in X$, there is $f \in L_{c}(X)$ such that $f$ belongs to no maximal ideal other than $M_{p}$ and $Z(f)=\{p\}$.

Proof. Take $f(x)=d(p, x)$. The proof is quite similar to (6.1).

Lemma 6.2. If $A(X)$ and $A(Y)$ satisfy (6-1), and $\Phi$ is an isomorphism from $A(X)$ onto $A(Y)$, then for any $M_{a} \subset A(X), \Phi\left(M_{a}\right)$ is a fixed maximal ideal in $A(Y)$. 
Proof. Consider the image of $f(x)=\sum_{i=1}^{n}\left(x_{i}-a_{i}\right)^{2}, \Phi(f)$. We can show that $Z(\Phi(f))=\{b\}$ for some $b \in Y$. The result follows immediately.

Lemma 6.2.' If $\Phi$ is an isomorphism of $L_{c}(X)$ onto $L_{c}(Y)$, then for $M_{p} \subset L_{c}(X), \Phi\left(M_{p}\right)$ is a fixed maximal ideal in $L_{c}(Y)$.

Proof is similar to $(6.2)$.

LemmA 6.3. Let $B_{1}$ and $B_{2}$ be subrings of $C(X)$ and $C(Y)$ respectively, which contain all constant functions, $\Phi$ be an isomorphism from $B_{2}$ to $B_{1}$, and $X$ be connected. Then $\Phi$ is the identity on the constant functions.

Proof. It is clear that $\Phi(r)=r$ for all rational constant functions $r$. If $k$ is an irrational number $k-r \neq 0$, for all rational numbers $r$. Moreover, $\Phi(k-r) \cdot \Phi(1 /(k-r))=\Phi(u)=u$, we have

$$
\Phi\left(\frac{1}{k-r}\right)=\frac{1}{\Phi(k)-r}
$$

for any rational number $r$. Suppose $\Phi(k)$ is not constant. By continuity of $\Phi(k)$ and connectedness of $X$, we would have $\Phi(1 /(k-r))$ is undefined for some $r$ and some point of $X$. This is a contradiction. Hence $\Phi(k)$ is constant. By $[3,0.22], \Phi$ is the identity on the constant functions.

THeORem 6.4. Let $X$ and $Y$ be two arbitrary subsets of $E^{n}$. If there are $A(x)$ and $A(Y)$ subrings of $C^{m}(X)$ and $C^{m}(Y)$ satisfying (6-1), and an isomorphism, $\Phi$, from $A(Y)$ onto $A(X)$ leaving all constant functions unchanged, then $\Phi$ induces a mapping $\tau: X \rightarrow Y$ defined by $\Phi(g)=g \cdot \tau$ and $\tau$ is a $C^{m}$-diffeomorphism.

Proof. Define $\tau$ to be a mapping from $X$ to $Y$ as follows: $\tau(x)=\cap Z\left[\Phi^{-1}\left(M_{x}\right)\right]$. By hypothesis and (6.2), $\Phi^{-1}\left(M_{x}\right)$ is a fixed maximal ideal in $A(Y)$. Thus, $\tau$ is well-defined. Evidently, $M_{\tau(x)}=$ $\Phi^{-1}\left(M_{x}\right)$, so that $\tau$ is one-one. Let $y_{0}$ be arbitrary in $Y$. Then $M_{y_{0}}$ is a fixed maximal ideal in $A(Y)$, and $\Phi\left(M_{y_{0}}\right)=M_{x_{0}}$ for some $x_{0} \in X$. Thus $y_{0}=\cap Z\left[\Phi^{-1}\left(M_{x_{0}}\right)\right]=\tau\left(x_{0}\right)$. That is, $\tau$ is onto. Now, for each $g \in A(Y)$ and each $x \in X$, if $\Phi(g)(x)=r$, then $\Phi(g)-r \in M_{x}, g-\Phi^{-1}(r) \in M_{\tau(x)}$, so that $g(\tau(x))=\left(\Phi^{-1}(r)\right)(\tau(x))=r(\tau(x))=r=\Phi(g)(x)$. Hence $\Phi(g)=$ $g \cdot \tau$. Similarly, $\Phi^{-1}(f)=f \cdot \tau^{-1}$ where $\tau^{-1}: Y \rightarrow X$, defined by $\tau^{-1}(y)=$ $\cap Z\left[\Phi\left(M_{y}\right)\right]$. Since $f \cdot \tau \in A(X)$ and $g \cdot \tau^{-1} \in A(Y)$ for each $g \in A(X)$ and $f \in A(Y)$, and $A(X)$ and $A(Y)$ contain all projections. By (5.4) $\tau$ is 
$C^{m}$-diffeomorphism.

Theorem $6.4^{\prime}$. Let $\left(X, d_{1}\right)$ and $\left(Y, d_{2}\right)$ be any two metric spaces. If there is an isomorphism $\Phi$ from $L_{c}(Y)$ onto $L_{c}(X)$ leaving all constant functions unchanged, then $\Phi$ induces a mapping $\tau: X \rightarrow Y$ defined by $\Phi(g)=g \cdot \tau$ and $\tau$ is an $L_{c}$-homeomorphism.

Proof is similar to $(6.4)$.

CoRollary 6.5. Let $X$ and $Y$ be two connected subsets of $E^{n}$. If there are subrings $A(X)$ and $A(Y)$ of $C^{m}(X)$ and $C^{m}(Y)$ respectively satisfying (6-1), and an isomorphism, $\Phi$, of $A(Y)$ onto $A(Y)$, then $\Phi$ induces $a C^{m}$-diffeomorphism, $\tau$, from $X$ onto $Y$ such that $\Phi(g)=g \cdot \tau$ for each $g \in A(Y)$.

Proof. Combine (6.3) and (6.4).

Corollary 6.5'. Let $\left(X, d_{1}\right)$ and $\left(Y, d_{2}\right)$ be two connected metric spaces. If $\Phi$ be an isomorphism of $L_{c}(Y)$ onto $L_{c}(X)$, then $\Phi$ induces an $L_{c}$-homeomorphism $\tau$ from $X$ on $Y$ such that $\Phi(g)=g \cdot \tau$ for each $g \in L_{c}(Y)$.

Proof is similar to $(6.5)$.

REMARK. In (6.4) if $A(X)=\mathscr{R}(X)$, and $A(Y)=\mathscr{R}(Y)$, then $\tau$ and $\tau^{-1}$ are not only $C^{m}$, each of their components is a rational function. We may name this mapping as rational-homeomorphism. We know that there is a nonlinear rational-homeomorphism. Let $X=Y=E^{n}-(0, \cdots, 0)$, and $\tau(x)=\left(\tau_{1}(x), \cdots, \tau_{n}(x)\right)$ be defined

$$
\tau_{i}(x)=\frac{x_{i}}{x_{1}^{2}+\cdots+x_{n}^{2}} \text { for } 1 \leqq i \leqq n
$$

Then its inverse is known to be $\tau^{-}(y)=\left(\Phi_{1}(y), \cdots, \Phi_{n}(y)\right)$ with

$$
\Phi_{j}(y)=\frac{y_{j}}{y_{1}^{2}+\cdots+y_{n}^{2}}, \quad 1 \leqq j \leqq n .
$$

If the metric spaces are compact subsets of $E^{n}$, then we have the same results as (6.1), (6.2), (6.4) and (6.5) with $A(X)$ and $A(Y)$ replaced by $B(X)$ and $B(Y)$ respectively, where $B(X)$ and $B(Y)$ are the subrings of $L(X)$ and $L(Y)$ respectively satisfying the following property: $\mathscr{R}(X) \subset B(X)$, and $f \in B(X)$ with $Z(f)=\Phi$ implies $1 / f \in B(X)$. We know that there is such a proper subring $B(X)$. For instance, let $B_{0}(X)=\left\{f \in L(X): f \in C^{3}(X)\right\}$. Then $\mathscr{R}(X) \subset B_{0}(X) \subset L(X)$. 
Next, we will see some algebraic properties of the rings of continuous functions which are inapplicable in the rings of $C^{m}$-differentiable function, where $1 \leqq m \leqq \infty$.

(1) The rings of $C^{m}$-differentiable functions are not lattice-ordered. Let $X=E^{1}$. Consider $C^{m}(X)$. We know $i(x)=x, i \in C^{m}(X)$. But $|i| \in C^{m}(X)$. Thus, neither $f \wedge \theta$ nor $f \vee \theta$, in general, is in $C^{m}(X)$.

(2) We know that in the rings of continuous functions, $I(f) \geqq 0$ if there is $g \in C(X)$ such that $g \geqq 0$ and $g \equiv f(\bmod I)$. (See [3,5.2 and 5.4(a)].) In the rings of differentiable functions such a $g$ need not exist. Consider $X=E^{1}$, and $C^{1}(X)$. Let $I=\left\{f \in C^{1}(X): Z(f) \supset[0,1]\right\}$. Then $I$ is a $z$-ideal, convex, but not absolutely convex. Let $f_{0}(x)=$ $x-x^{2}$. It is clear that $f_{0} \geqq 0$ on a zero-set of $I$. But there is no $g \in C^{1}(X)$ so that $g \geqq 0$ and $g$ agrees with $f_{0}$ on $[0,1]$.

(3) If $I$ and $J$ are $z$-ideals in $C(X)$, then $I J=I \cap J$. This is not true in $C^{m}(X)$. Let $X=E^{1}$. Consider in $C^{1}\left(E^{1}\right), I=J=M_{0}=$ $\left\{f \in C^{1}\left(E^{1}\right): f(0)=0\right\}$. Then $I \cap J=M_{0}$. But $i(x)=x, i \in I \cap J$ and $i \notin I J$. This also shows that the following is not true in $C^{m}(X)$ or $C^{m *}(X)$. If $P$ and $Q$ are prime ideals in $C$ (or $C^{*}$ ) then $P Q=P \cap Q$. For $C^{m *}$, we take $X=(-n, n)$.

\section{REFERENCES}

1. R. Bkoucke, Ideaux mous d'un anneau commutatif application aux anneaux de functions, C. R. Acad. Sc. Paris 260 (1965), 6496-6498.

2. R. M., Crownover, Concerning function algebras, Studia Math. 25 (1965), 353-365.

3. L. Gillman and M. Jerison, Rings of continuous functions, D. Van Nostrand, New Jersey, 1960.

4. E. Hewitt, Rings of real-valued continuous functions I, Trans. Amer. Math. Soc. 64 (1948), 45-99.

5. J. G. Hocking and G. S. Young, Topology, Addison-Wesley, Massachusetts, 1961.

6. K. D. Magill, Jr., Some embedding theorems, Proc. Amer. Math. Soc. 16 (1965), 126-130.

7. E. J. McShane, and T.A. Botts, Real Analysis, D. Van Nostrand, Toronto, 1959.

8. J., Milnor, Differential Topology, Princeton Univ. Press, 1958.

9. S. B. Myers, Algebras of differentiable functions, Proc. Amer. Math. Soc. 5 (1964), 917-922.

10. M. Nakai, Algebras of some differentiable functions on Riemannian manifolds, Japan J. Math. 29 (1959), 60-67.

11. L. E. Pursell, An algebratic characterization of fixed ideals in certain function rings, Pacific J. Math. 5 (1955),

12. D. R. Sherbert, Banach algebras of Lipschitz functions, Pacific J. Math. 13 (1963), 1387-1399.

13. M. H. Stone, Applications of the theory of Boolean rings to general topology, Trans. Amer. Math. Soc. 41 (1937) 375-481.

14. H. Whitney, Analytic extension of differentiable function defined in closed sets, Trans. Amer. Math. Soc. 36 (1934), 63-89.

15. - Differentiable functions defined in arbitrary subsets of Euclidean space, Trans. Math. Soc. 40 (1936), 309-317.

16. - Differentiable Manifolds, Ann. of Math. 37 (1936), 645-680. 
Received February 21, 1967. This paper is a portion of the author's dissertation submitted as a partial requirement for the $\mathrm{Ph}$. D. degree at the University of British Columbia under the direction of Professor James V. Whittaker to whom the author is very much indebted. Research was supported by Canadian National Research Council Studentship and Assistantship of U.B.C.

DEPARTMENT OF MATHEMATICS

THE UNIVERSITY OF OKLAHOMA 



\section{PACIFIC JOURNAL OF MATHEMATICS}

EDITORS

H. ROYDEN

Stanford University

Stanford, California

R. R. Phelps

University of Washington

Seattle, Washington 98105
J. DugundJI

Department of Mathematics

University of Southern California

Los Angeles, California 90007

\section{RICHARD ARENS}

University of California

Los Angeles, California 90024

\section{ASSOCIATE EDITORS}
E. F. BeCKENBACH
B. H. NeUmanN
F. WOLF
K. YOSIDA

\section{SUPPORTING INSTITUTIONS}

UNIVERSITY OF BRITISH COLUMBIA CALIFORNIA INSTITUTE OF TECHNOLOGY UNIVERSITY OF CALIFORNIA MONTANA STATE UNIVERSITY UNIVERSITY OF NEVADA NEW MEXICO STATE UNIVERSITY OREGON STATE UNIVERSITY UNIVERSITY OF OREGON OSAKA UNIVERSITY UNIVERSITY OF SOUTHERN CALIFORNIA
STANFORD UNIVERSITY UNIVERSITY OF TOKYO UNIVERSITY OF UTAH WASHINGTON STATE UNIVERSITY UNIVERSITY OF WASHINGTON

AMERICAN MATHEMATICAL SOCIETY CHEVRON RESEARCH CORPORATION TRW SYSTEMS NAVAL WEAPONS CENTER 


\section{Pacific Journal of Mathematics \\ Vol. 27, No. $1 \quad$ January, 1968}

Willard Ellis Baxter, On rings with proper involution ............... 1

Donald John Charles Bures, Tensor products of $W^{*}$-algebras........... 13

James Calvert, Integral inequalities involving second order derivatives . . . . 39

Edward Dewey Davis, Further remarks on ideals of the principal class.... 49

Le Baron O. Ferguson, Uniform approximation by polynomials with integral

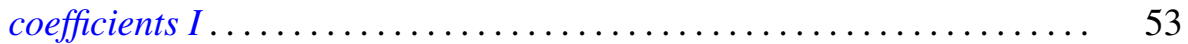

Francis James Flanigan, Algebraic geography: Varieties of structure

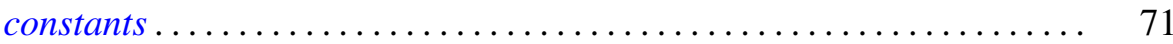

Denis Ragan Floyd, On QF -1 algebras ..................... 81

David Scott Geiger, Closed systems of functions and predicates ......... 95

Delma Joseph Hebert, Jr. and Howard E. Lacey, On supports of regular Borel measures ................................... 101

Martin Edward Price, On the variation of the Bernstein polynomials of a function of unbounded variation ........................ 119

Louise Arakelian Raphael, On a characterization of infinite complex matrices mapping the space of analytic sequences into itself........ 123

Louis Jackson Ratliff, Jr., A characterization of analytically unramified

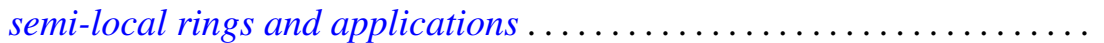

S. A. E. Sherif, A Tauberian relation between the Borel and the Lototsky transforms of series ................................ 145

Robert C. Sine, Geometric theory of a single Markov operator .......... 155

Armond E. Spencer, Maximal nonnormal chains in finite groups......... 167

Li Pi Su, Algebraic properties of certain rings of continuous functions .... 175

G. P. Szegô, A theorem of Rolle's type in $E^{n}$ for functions of the class $C^{1} \ldots 193$

Giovanni Viglino, A co-topological application to minimal spaces ........ 197

B. R. Wenner, Dimension on boundaries of $\varepsilon$-spheres ............... 201 